\title{
Impact of Modulation Schemes on LTE
}

\author{
Juhi Pruthi \\ IIIT Delhi
}

\author{
Pooja Agarwal \\ IIIT Delhi
}

\section{Nikita Jain IIIT Delhi}

\begin{abstract}
Today, mobile communications plays a central role in the voice/data network arena. With the deployment of mass scale $3 \mathrm{G}$ around the corner, new directions are already being researched. This paper presents glimpse of LTE an evolving $4 \mathrm{G}$ technology in cellular world. In order to enhance its performance in future, it's imperative to improve upon the transmitted signal strength. Consequently, analysis of the existing modulation schemes becomes a necessity in order to integrate them with transmitted signal.This provides a platform to facilitate better transmission of carrier signal with maximum reliability and less attenuation. Thus resulting in maximum throughput and high bit rates in LTE network. We therefore propose a novel extension of these modulation schemes to LTE-Advanced in context of relay nodes.
\end{abstract}

\section{Keywords}

Donor NodeB(deNB), enodeB(eNB), Quadrature Amplitude Modulation(QAM), Quadrature Phase Shift Keying (QPSK), Relay Node(RN)

\section{INTRODUCTION}

The main objective of LTE network is to provide higher capacity with high bitrates and reduce interference amongst users. This motivates the concept of simulating and analyzing the impact of modulation schemes on throughput and call drop probability due to interference amongst multiple users.Optimum modulation scheme having a balance of interference and bit-rate must be implemented.This leads to the proposal of an envisage framework of integrating modulation schemes into the relay nodes.

Cellular network is a radio-based technology. Radio waves are electromagnetic waves that antennas propagate.

It is useful to think of cellular Network in terms of generations: [1]

-0G:Briefcase-size mobile radio telephones: PTT(Push to Talk ), MRT(Mobile Radio Telephone)

-1G:Analog cellular telephony: AMPS(Advanced Mobile System used in North America)

$-2 \mathrm{G}$ :Digital cellular telephony

-3G:High-speed digital cellular telephony (including video telephony)

-4G:Internet facility: anytime, anywhere voice, data, and multimedia telephony at faster data rates than $3 \mathrm{G}$

The above stated is shown in figure 1 .

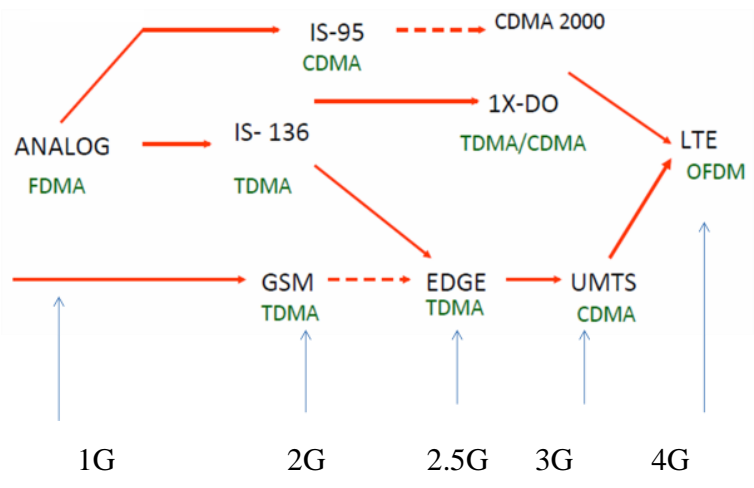

Fig 1: Evolution of cellular network

Mobile radio technology has evolved from time-division multiplexing (TDM) and code-division multiplexing (CDM) to orthogonal frequency-division multiplexing (OFDM),which offers improved spectral efficiency by integrating orthogonal carriers. OFDM is deployed by $4 \mathrm{G}$ networks, such as Long Term Evolution (LTE) and WiMAX.In LTE-Advanced focus is on higher capacity. The main driving force to further develop LTE towards LTEAdvanced was to provide higher bitrates in a cost effective way[1].

\subsection{Modulation}

In a carrier communication system, the baseband signal of a low-frequency spectrum is translated to a high frequency spectrum. This is achieved through modulation. The aim of this topic is to explore the reasons for using modulation. Modulation is defined as a process by which certain parameter like amplitude of a high frequency sinusoidal wave is varied in accordance with the instantaneous magnitude of the baseband signal[2].

Two signals are involved in the modulation process. The baseband signal and the carrier signal. The baseband signal is to be transmitted to the receiver. The frequency of this signal is generally low. In the modulation process, baseband signal is called the modulating signal. The waveform of baseband signal is unforeseeable.For example, the waveform of a speech signal (modulating signal) is random and arbitrary in nature.It varies the amplitude or phase or both of the carrier signal[2].

Practically, it has been concluded that [3] :

- Higher order modulation schemes are less resilient to noise and interference.

- On the contrary, low order modulation schemes are more reliable with fewer errors and re-sends.

There exist various modulation schemes as illustrated in table $1[3]$. 
Table 1: Modulation Schemes

\begin{tabular}{|c|c|c|}
\hline Scheme & $\begin{array}{c}\text { Bits per } \\
\text { Symbol }\end{array}$ & Symbol Rate \\
\hline QPSK & 1 & $1 / 1$ bit rate \\
\hline BPSK & 2 & $1 / 2$ bit rate \\
\hline 8PSK & 3 & $1 / 3$ bit rate \\
\hline 16QAM & 4 & $1 / 4$ bit rate \\
\hline 64QAM & 6 & $1 / 6$ bit rate \\
\hline
\end{tabular}

Following this introduction ,the paper is organized as follows. Section 2 describes LTE(4G), section 3 describes LTE-Advanced.Section 4 presents insight into analysis and findings and finally section 5 describes conclusion.

\section{LTE (4G)}

LTE (Long Term Evolution)is successor to $3 \mathrm{G}$ with functionalities of nodeB and BTS combined together as enodeB.

EnodeB is termed as or the E-UTRAN(Evolved Universal Terrestrial Access Network) since it supports both circuitswitched and packet-switched connections.The main requirements for the new access network are high spectral efficiency, high peak data rates, short round trip time as well as flexibility in frequency and bandwidth[4].LTE architecture is specified in figure 2 .

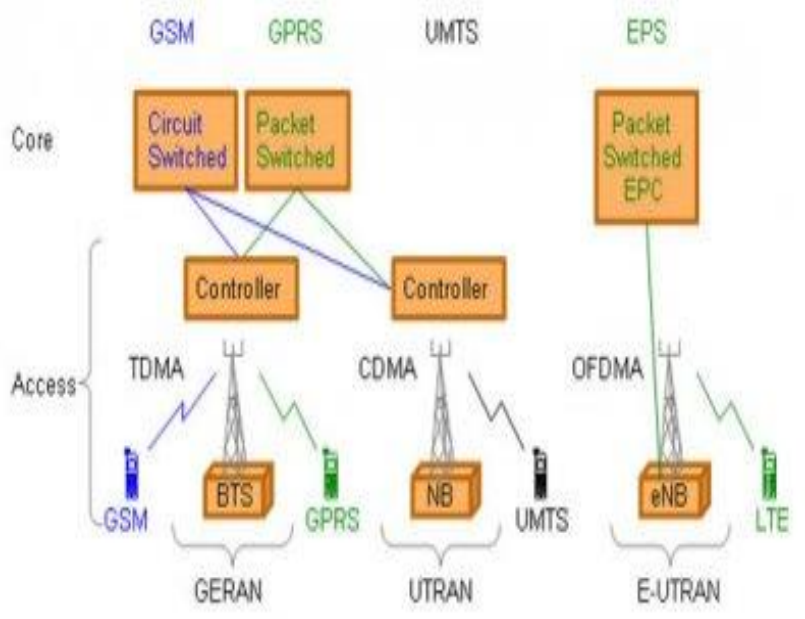

Fig 2: LTE architecture

GSM was developed to carry real time services basically data services over dedicated circuit switched connection with very low data rates. The Evolved Packet Core(EPC) carries circuit switched as well as IP traffic,that is both real time services and datacom services[4].

HeNBs are introduced mainly to provide coverage indoors, in homes or offices.It incorporates the capabilities of nodeB and resource management feature of radio network controller. Normally it is owned by the customer and connects to the operators EPC(Evolved Packet Core)[4].Arrangement of femtocell and macrocell within a cell zone is shown in figure 3.

\section{b) Home eNB (HeNB)}

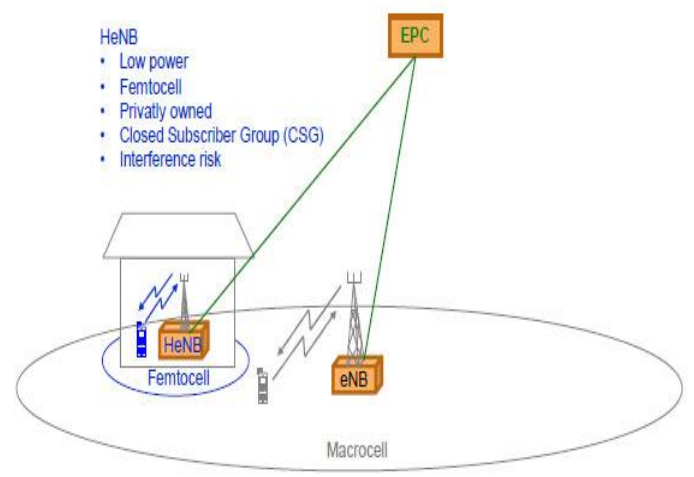

Fig 3: Home NodeB(HeNB)

\section{RELAY NODE (RN)}

LTE-Advanced supports a feature called Relay node(RN).RN are low power eNodeB's that provide enhanced coverage and system capacity at the cell boundaries.It also supports remote areas where there is no fiber connection.It has a striking feature i.e supporting the eNB functionality as well as subset of the UE functionality. It connects wirelessly to the DeNB using $U n$ interface. DeNB shares its radio resources with the $\mathrm{RN}[5]$. The architecture for relay node is shown in figure 4 [6].

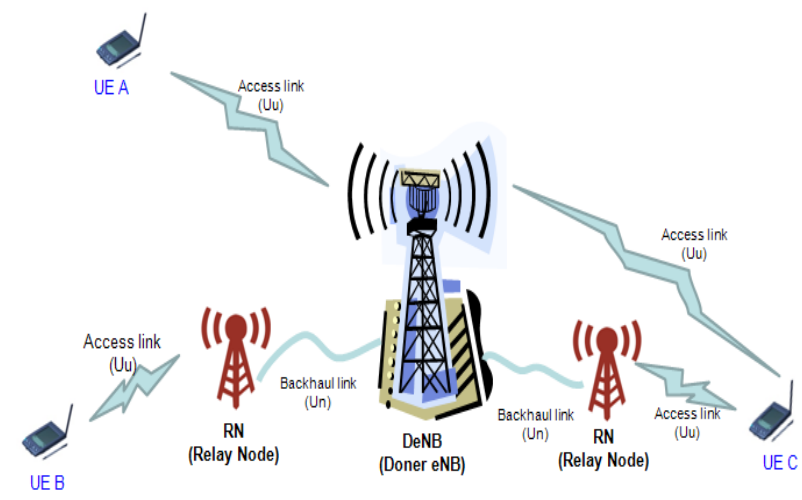

Fig 4: LTE-Advanced architecture

\subsection{Transmission Schemes}

Many transmission schemes have been proposed to establish two-hop communication between an DeNB and UE through RN.

- Amplify and Forward(AF) - RN receives the signal from the eNB (or UE) at the first phase. It amplifies the received signal and forwards it to the UE (or eNB) at the second phase.This scheme is very simple and has very short delay.However,it also amplifies the noise[7].

- $\quad$ Selective Decode and Forward $(D F)$ - RN decodes (channel decoding) the received signal from the eNB (UE) at the first phase. If the decoded data is correct using cyclic redundancy check (CRC), the RS will perform channel coding and forward the new signal to the UE (eNB) at the second phase. This DCF scheme can effectively avoid error 
propagation through the $\mathrm{RN}$, but the processing delay is quite long [7].

- Compress and Forward - Compress and Forward is similar to the previously mentioned protocol apart from the implementation where the relay node compresses and then transmits the received signal to the destination[7].It outperforms DF when the link between the relay and destination is better than that between the source and relay [8]

\section{SIMULATION AND ANALYSIS}

Femto-cells have a circular area, each with a fixed radius. A fixed number of macro users and femto users i.e. 5 and one FBS are uniformly distributed in the femto cell. Objective is to find how macro users and femto users are effected by modulation schemes and how the outage probability varies for each of them.

\subsection{Single Frequency Band vs Modulation Schemes}

For a constant frequency of $20 \mathrm{MHz}, 64 \mathrm{QAM}$ gives better throughput upto a distance of $60 \mathrm{kms}$. For distances above $60 \mathrm{kms}, 8 \mathrm{PSK}$ outperforms 64QAM.

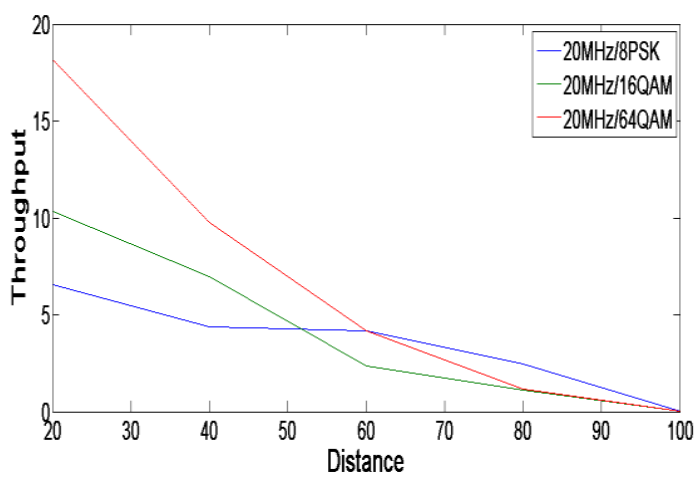

Fig 5: Comparison of modulation schemes on single frequency band

\subsection{Multiple Frequency Bands vs Single Modulation Scheme}

Objective of LTE is to provide high bit rates, so chosen scheme is 64QAM since it has the highest bit rate against multiple frequency bands.From figure 6 finding is $20 \mathrm{MHz}$ performs the best amongst all frequency bands.

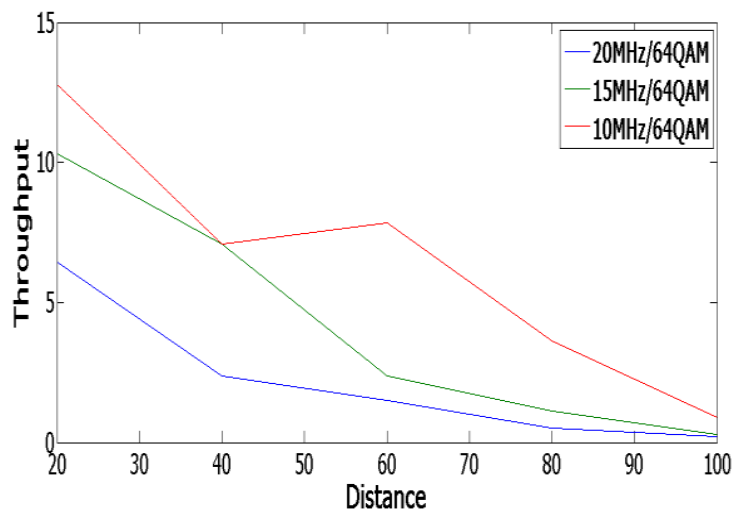

Fig 6: Comparison of modulation scheme on multiple frequency bands

\subsection{Throughput vs Modulation Scheme}

Aim is to find how throughput varies for increased number of users within a cell being served by single femto base station.

\subsubsection{QAM}

Figure 8 depicts that as number of femto users increases throughput keeps decreasing.For large distances almost approaches 0 .

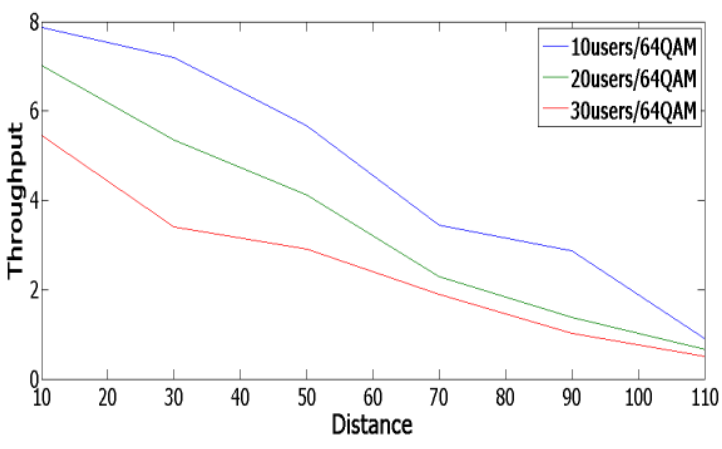

Fig 8: Comparison of throughput for increasing number of users

\subsection{2 $8 P S K$}

Figure 9 depicts throughput is inversely proportional to number of users. Also throughput of 8PSK is very low as compared to 64QAM from figure 8 .

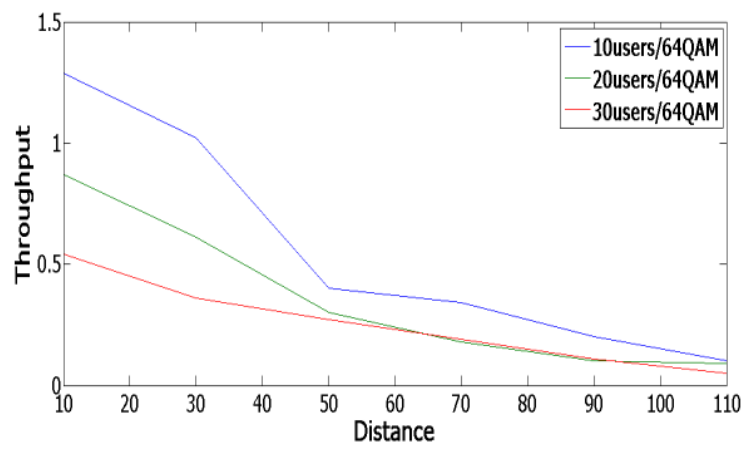

Fig 9: Comparison of throughput for increasing number of users

\subsection{Outage Probability vs Modulation \\ Scheme}

Outage probability requires finding call drop probability which is effected by signal-to-interference ratio.

Figure 10 shows high signal-to-inference ratio for QAM due to which call drops of various users increases, hence outage probability gets increased.

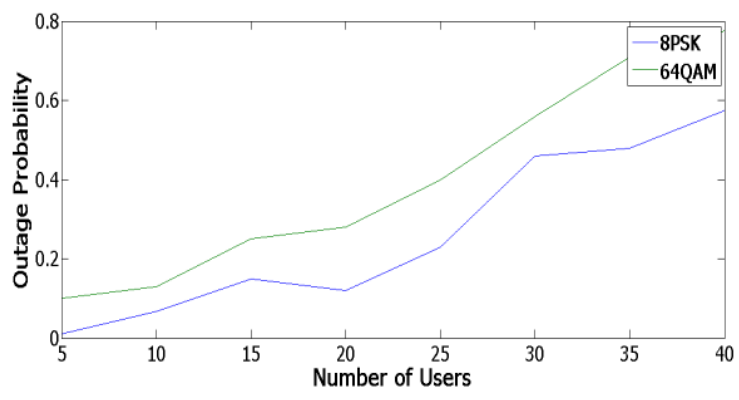

Fig 10:Outage probability versus number of users 


\section{PROPOSED FRAMEWORK}

We propose the extension of modulation schemes to the relay nodes in context of three transmission schemes:

- Amplify and Forward- both the DeNB and RN should deploy 8PSK modulation as in this transmission scheme alongwith the signal, noise is also amplified.From simulations it is depicted that 8PSK being resilient to noise shows low outage probability and will result in better signal transmission in such scenario.

- Selective Decode and Forward- RN should deploy 8PSK and DeNB should deploy 64QAM. Since relay node performs crc check 8PSK will result in less processing delay due to less inherited noise.Whereas DeNB should use 64QAM as it receives error-detected signal from relay node which is more reliable,so high data rate is the main objective.

- Compress and Forward- in this scheme 8PSK should be implemented as it is resilient to noise and will result in less propagation delay as compared to 64QAM which is more prone to interference and will result in lot of retransmissions due to $\mathrm{CRC}$ check by $\mathrm{RN}$.

\section{CONCLUSION}

Optimal Implementation -relay node should be integrated with modulation scheme which is an efficient combination of reliability and high-transmission speed.

So an optimal choice would be to use $8 P S K$ in all three transmission schemes.In addition to ensure high data rate,we can integrate $8 P S K$ with padding bits so as to maintain low power requirements within constrained bandwidth and to ensure short delays, we can use small interleaving periods.

Also we can deploy $64 Q A M$ modulation scheme alongwith low-density parity check(ldpc) as ldpc outperforms turbo coding in scenarios using high-data rates.Low-density paritycheck $(L D P C)$ code is a linear error correcting code, a method of transmitting a message over a noisy transmission channel. It decreases the complexity of the encoder with increasing data rate as it consumes less cycles and gives faster results. LDPC codes are finding increasing use in applications requiring reliable and highly efficient information transfer over constrained bandwidth links in the presence of corrupting noise[9].

\section{REFERENCES}

[1] TheMobileBroadbandStandard,3GPP

[2] DAENotes,Need For Modulation

[3] Radio-electronics.com:quadrature-amplitudemodulation

[4] LTE,3GPP

[5] Relay Nodes (Stefania Edition 2011)

[6] LTE_Advanced_R10_Network_Overview

[7] P.Thenkumari,Power Control Optimization and Improving Throughput for LTE-Advanced Relay Networks, International Journal of Engineering and Innovative Technology (IJEIT),Volume 3, Issue 2, August 2013, ISSN: 2277-3754 , ISO 9001:2008 Certified, in press.

[8] J. Jiang,J.S. Thompson, P.M. Grant, and N. Goertz,Practical Compress and Forward Cooperation for the Classical Relay Network,17th European Signal Processing Conference (EUSIPCO 2009) Glasgow, Scotland, August 24-28, 2009

[9] Wikipedia, Low-densitys_parity-check_code 